\title{
Refractory hyperkalaemia associated with trimethoprim-sulfamethoxazole therapy in neutropaenic sepsis
}

\author{
ME Maddumage ${ }^{1^{*}}$, AYA Gunasekara ${ }^{2}$, WR Samaranayake ${ }^{3}$ \\ Consultant Intensivist ${ }^{l}$, Medical Officer ${ }^{2}$, Senior Registrar in Critical Care Medicine ${ }^{3}$, Medical \\ Intensive Care Unit, National Hospital of Sri Lanka, Sri Lanka.
}

\begin{abstract}
Hyperkalaemia is a potentially life threatening electrolyte disorder commonly seen in critically ill patients secondary to renal impairment. Herein, we report a case of severe hyperkalaemia, which was disproportionate to the degree of renal impairment, associated with neutropaenic sepsis, in a critically ill middle aged gentleman. Hyperkalaemia was refractory to medical treatment necessitating haemodialysis in the background of recent administration of trimethoprim- sulfamethoxazole (TMX-SMX) for presumed pneumocystis jiroveci pneumonia. It is important for clinicians to be aware of this complication as TMX-SMX is a popular antibiotic in immunocompromised populations.
\end{abstract}

Keywords: hyperkalaemia; trimethoprim- sulfamethoxazole; leukaemia; neutropaenic sepsis; haemodialysis; pneumocystis jiroveci pneumonia

\section{Case presentation}

A 58yr old caucasian man was admitted to intensive care due to type 1 respiratory failure associated with febrile neutropaenia. He was diagnosed as having acute lymphoblastic leukaemia [ALL-Philadelphia Ch negative) 12 months ago, and developed liver toxicity following induction with UKALL 14 protocol of chemotherapy. ${ }^{1}$ Consequently, he was switched to UKALL 60+ protocol upon recovery of hepatic function, after 8 weeks. He was further diagnosed as having type 2 diabetes mellitus (T2DM) 11 months ago, and achieved satisfactory glycaemic control with metformin therapy. He had a single relapse of ALL after 6 months of starting chemotherapy, but otherwise remained in biopsyproven remission. His course of the illness was further complicated by several episodes of febrile neutropaenia including this admission. His leukocyte count (WCC) was $0.27 \times 10^{9} / \mathrm{L}$ on admission and bilateral infiltrates were present on chest X-ray suggestive of severe pneumonia (Figure 1).

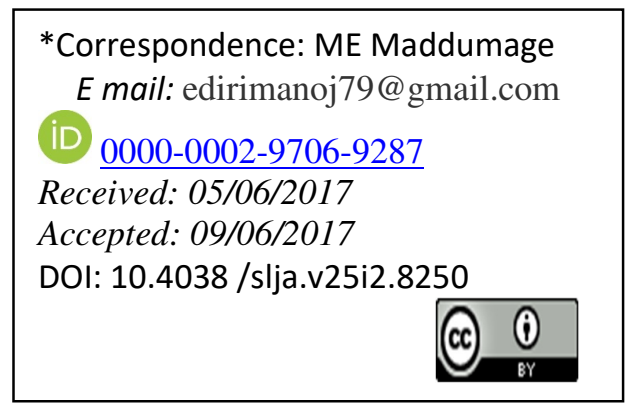

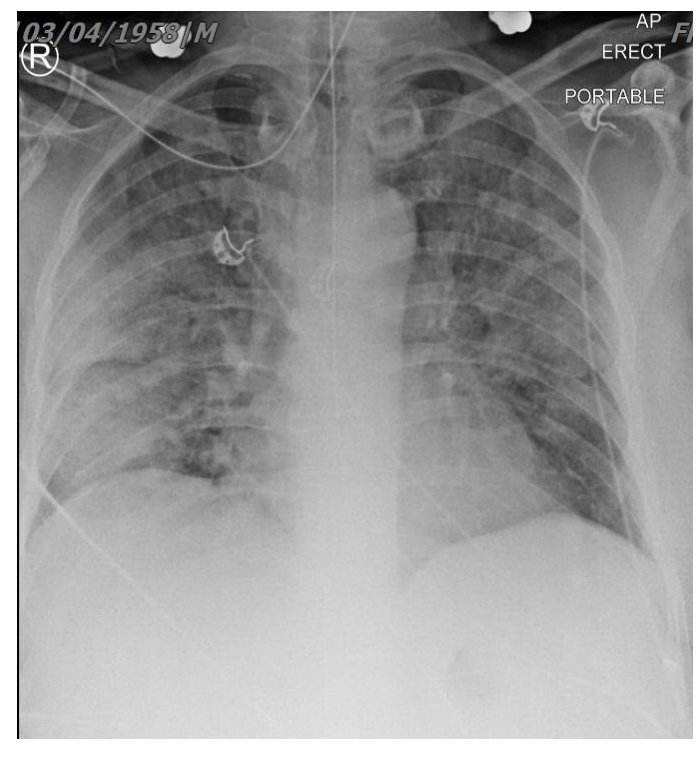

Figure 1: Bilateral infiltrates in both lung fields suggestive of ARDS

Respiratory failure rapidly progressed inspite of intravenous meropenem, clarithromycin and granulocyte colony stimulating factor (GCSF) which were started on admission. He was further started on trimethoprim-sulfamethoxazole (TMX-SMX) empirically at a dose of $2.4 \mathrm{~g} 6 \mathrm{hrly}$ along with $60 \mathrm{mg}$ prednisolone daily upon admission to the intensive care to cover presumed pneumocystis jiroveci pneumonia. (PJP) $\mathrm{He}$ initially had non-invasive respiratory support for $48 \mathrm{hrs}$ but escalated to intermittent positive pressure ventilation due to rapid deterioration of oxygenation with evidence of severe acute 
respiratory distress syndrome (ARDS). He further had prone ventilation $18 \mathrm{hrs}$ later as ventilation was difficult despite high level of sedation with $1 \%$ propofol $(350 \mathrm{mg} /$ hour $)$, high support pressures, other recruitment manoeuvres and muscle paralysis with cis-atracurium intravenous infusion. His lung mechanics and oxygenation showed a gradual progress and he was turned supine following $9 \mathrm{hrs}$ of prone ventilation. His neutropaenia further worsened and TMX-SMX was stopped after 5 days, as the bronco-alveolar lavage (silver stain) did not reveal obvious evidence of pneumocystis jiroveci infection. His steroid dose was planned for gradual tapering. On day 6 morning, his serum potassium was detected to be high $(6.4 \mathrm{mmol} / \mathrm{L})$ despite a good urine output and mild deterioration of renal function (estimated glomerular filtration rate (eGFR) $52 \mathrm{mls} / \mathrm{min} / 1.73 \mathrm{~m}^{2}$ ). His arterial blood gases showed respiratory acidosis which was partially compensated metabolically $(\mathrm{pH}$ 7.27, $\mathrm{PCO}_{2} 6.2 \mathrm{KPa}, \mathrm{PO}_{2} 12 \mathrm{KPa}, \mathrm{BE} 2, \mathrm{HCO}_{3}$ $27 \mathrm{mmol} / \mathrm{L}$ ). Neither his creatinine kinase (CK) nor serum uric acid levels were elevated. Hyperkalaemia was refractory to medical management and needed continuous veno-venous haemodialysis (CVVHD) at a dose of $25 \mathrm{ml} / \mathrm{kg} / \mathrm{h}$ for the management of life threatening hyperkalaemia. (7.6mmol/L) His serum potassium normalised after $72 \mathrm{hrs}$ of CVVHD and he remained without renal support thereafter. He had a failed extubation 9 days after invasive ventilation associated with severe muscle weakness suggestive of critical illness neuromyopathy (CINM). Subsequently, he underwent percutaneous tracheostomy and was decannulated after a further 10 days of gradual weaning from ventilatory support. Six days after stopping TMX-SMX, his PJP PCR became positive and TMX-SMX was restarted. Strangely, hyperkalaemia did not recur and neutropaenia continued to improve. He was discharged to the ward after 22 days of intensive care.

\section{Discussion}

Hyperkalemia is a critical condition, potentially fatal with cardiac dysrhythmias. Hyperkalemia in intensive care is most often linked to renal impairment, but may be associated with many other conditions including: rhabdomyolysis, adrenal dysfunction, medications, tumour lysis syndrome (TLC), propofol infusion syndrome (PIS), ongoing haemolysis, pseudo hyperkalaemia and type 4 renal tubular acidosis
(RTA). Our patient, was critically ill having neutropaenic sepsis with ARDS and almost all the causes for hyperkalaeemia mentioned above were relevant and needed exclusion.

Different blood samples taken from his arterial line and central venous line indicated that our patient had true hyperkalaemia along with corresponding ECG changes. The hyperkalaemia was essentially out of proportion to the mild degree of AKI with eGFR $52 \mathrm{mls} / \mathrm{min} / 1.73 \mathrm{~m}^{2}$, and further worsened despite improving renal functions. Adrenal dysfunction was considered as a potential cause for his hyperkalamia in the background of high dose steroids which was subsequently tapered appropriately. He neither had clinical nor other biochemical manifestations to make adrenal insufficiency the cause of hyperkalaemia. Evidence for rhabdomyolysis or haemolysis was absent. Absence of metabolic acidosis or bradyarrhythmias along with normal CK despite heavy sedation with propofol (3.3 $\mathrm{mg} / \mathrm{kg} / \mathrm{hr}$ ) was not suggestive of PIS. Normal uric acid level and sustained remission of ALL made TLC unlikely. Type 4 RTA which is known to be associated with T2DM, was unlikely in this gentleman who had no evidence of end organ damage but achieved a reasonable glycaemic control during a short period of T2DM. We could not find any obvious medication in his prescription i.e: potassium supplements, angiotensin-converting enzyme inhibitors (ACEi), potassium-sparing diuretics or heparin which are commonly known drugs leading to hyperkalaemia . Interestingly, TMX-SMX, which was stopped $24 \mathrm{hrs}$ before he started developing hyperkalaemia, was presumed to be the only culprit accountable for this catastrophe.

Most of the earliest cases of trimethopriminduced hyperkalemia were reported in patients with HIV/AIDS. These patients are typically treated with large doses of TMX-SMX for pneumocystis jiroveci. ${ }^{2}$ More recent clinical studies have shown that hyperkalemia can be seen even with "standard-dose" TMX-SMX. ${ }^{3}$ However infrequent the occurrence, it is important to understand the mechanism by which the adverse effect occurs. Trimethoprim has weakly basic properties due to positively charged and weakly basic amino groups attached to heterocyclic ring structures, which makes them similar in structure to the widely-used potassiumsparing diuretics amiloride and triamterene. 
Amiloride blocks the epithelial sodium ion channel $\mathrm{ENaC}$, which is responsible for sodium reabsorption in the distal nephron of the kidney. ${ }^{4} \mathrm{ENaC}$ also indirectly controls potassium secretion and excretion in the urine, and it is stimulated by aldosterone.

\section{Conclusion}

It is not infrequent for intensive care physicians to encounter unexpected life threatening complications in critically ill patients. Prompt decision making and aggressive supportive therapy is pivotal, even when the obvious culprit, compounding the clinical course is illusive. Due to its popularity in the medical field and to the largely unrecognized effect of hyperkalemia, it is important to consider such adverse effects when prescribing TMX-SMX. On the other hand, this case illustrates the management of an extremely critically ill patient with neutropaenic sepsis, ARDS, CIPM and difficult weaning from ventilator support towards a favourable outcome.

\section{References}

1. Guideline For The Management Of Acute Lymphoblastic Leukaemia (ALL) Version 20. Doc

2. Mihm LB, Rathbun RC, Resman - Targoff BH. Hyperkalemia associated with high-dose trimethoprim - sulfamethoxazole in a patient with the acquired immunodeficiency syndrome. Pharmacotherapy 1995;15(6):793- 797. PMid:8602391

3. Perazella MA, Mahnensmith RL. Trimethoprimsulfamethoxazole: hyperkalemia is an important complication regardless of dose. Clinical Nephrology 1996;46(3):187- 192.

PMid:8879854

4. Perazella MA. Trimethoprim-induced hyperkalaemia. Clinical data, mechanism, prevention and management. Drug Safety 2000; 22(3): 227-236. https://doi.org/10.2165/00002018-200022030$\underline{00006}$

PMid: 10738846 